\title{
Zu viel Salz erhöht das Risiko bei Hypertonikern - zu wenig bei allen
}

\author{
Studien konnten eine U-förmige Assoziation zwischen Natriumaufnahme und kardiovaskulä- \\ ren Ereignissen bzw. Tod zeigen. Demnach besteht sowohl bei einer geringen als auch bei \\ einer hohen Natriumaufnahme ein erhöhtes kardiovaskuläres Risiko. Ob diese Assoziation \\ durch den Hypertoniestatus der Patienten beeinflusst wird, haben Forscher nun untersucht.
}

Die Analyse basierte auf Daten von 4 großen Studien mit insgesamt 133118 Individuen aus 49 Ländern. 63559 Studienteilnehmer litten unter hohem Blutdruck. Die 24-stündige Natriumexkretion wurde als Maß für die Natriumaufnahme auf Gruppenniveau erfasst und auf den kombinierten Endpunkt aus Tod und schweren kardiovaskulären Ereignissen sowie den Blutdruck bezogen.

Ein erhöhter Natriumkonsum führte zu einer stärkeren Erhöhung des systolischen Blutdrucks bei Hypertonikern als bei Teilnehmern ohne Bluthochdruck (Änderung pro Gramm Natriumzunahme $2,08 \mathrm{mmHg}$ vs. 1,22 mmHg; $\mathrm{p}_{\text {Interaktion }}<0,0001$ ). Innerhalb der Hypertonie-Gruppe war sowohl eine Natriumexkretion von $7 \mathrm{~g} / \mathrm{Tag}$ oder mehr als auch von weniger als $3 \mathrm{~g} / \mathrm{Tag}$ (jeweils $11 \%$ aller Hypertoniker; $p<0,0001$ ) mit einem erhöhten Risiko im Vergleich zu 4-5g/Tag Natriumausscheidung assoziiert (25\% aller Hypertoniker). Bei Normotonikern erhöhte eine stärkere Natriumexkretion im Vergleich $\mathrm{zu}$ einer Ausscheidung von 4-5g/Tag (27\% aller Normotoniker) das Risiko für den primären kombinierten Endpunkt nicht $(\geq 7 \mathrm{~g} /$ Tag bei $9 \%$ aller Normotoniker; $\mathrm{p}=0,2547)$. Im Gegensatz dazu war bei dieser Gruppe eine Natriumexkretion von weniger als $3 \mathrm{~g}$ / Tag mit einem signifikant erhöhten Risiko für den kombinierten Endpunkt assoziiert (11\% aller Normotoniker; $\mathrm{p}=0,0009$ ).

Die Autoren empfehlen deshalb, die Kochsalzzufuhr nur bei Patienten mit Bluthochdruck und erhöhter Natriumaufnahme zu beschränken. Dies betraf etwa $10 \%$ der untersuchten Studienteilnehmer.

Dr. rer. nat. Frank Lichert, Weilburg

DOI 10.1055/s-0042-117074

Mente et al. Associations of urinary ...

Lancet 2016; 388: 465-475

\section{Kommentar aus der Praxis}

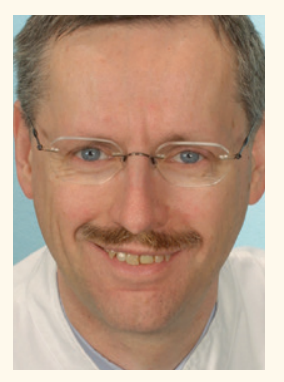

Prof. Dr. med. Walter Zidek

Die aktuelle Publikation bestätigt erneut den Zusammenhang zwischen einer erhöhten Kochsalzzufuhr und kardiovaskulären Erkrankungen. Eine besondere Stärke der Publikation ist die enorme Größe der Datenbasis. Umgekehrt ist es bei so großen Probandenzahlen naturgemäß sehr schwierig, die Kochsalzausscheidung genau zu messen. Daher arbeiten diese großen epidemiologischen Studien mit einer Schätzung, die allerdings eine gewisse Ungenauigkeit bedingt.

Die gesonderte Analyse von Hypertonikern und Normotonikern bestätigt die Erfahrung, dass das Risiko durch erhöhte Kochsalzzufuhr nur bei erhöhtem Blutdruck besteht. Zum wiederholten Mal wurde auch nachgewiesen, dass eine besonders niedrige Kochsalzzufuhr das Risiko steigert, ein schweres kardiovaskuläres Ereignis zu erleiden oder zu versterben. Hier besteht interessanterweise kein Unterschied zwischen Hypertonikern und Normotonikern.

Bei der Interpretation dieses Befundes ist sicher auch das Problem der soge- nannten „reverse epidemiology“ zu bedenken: Dies bedeutet, dass Menschen, denen es aus unterschiedlichsten Gründen sehr schlecht geht (konsumierende Erkrankungen, schwere Herzinsuffizienz, Demenz u.a.) bei den meisten Risikofaktoren (z.B. Kochsalzzufuhr, Cholesterin, Body Mass Index) besser abschneiden als der Durchschnitt. Der Grund ist einfach der, dass mit der verminderten Nahrungszufuhr auch die Zufuhr von Kochsalz, tierischen Fetten und Kalorien geringer ist. Dennoch ist selbstverständlich die Prognose dieser Gruppe besonders schlecht.

Ich wäre also mit der Schlussfolgerung vorsichtig, dass eine besonders niedrige Kochsalzzufuhr ungesund sein könnte. Die Kochsalzrestriktion bei Hypertonie erfährt hingegen eine erneute Bestätigung. Die seit Jahren geführten Debatten um den Wert der Kochsalzrestriktion haben ohnedies mehr den Charakter von Scheingefechten, seitdem die Evidenz im Sinne einer Kochsalzbeschränkung immer eindeutiger geworden ist. Für die Praxis bedeutet dies, dass wir weiterhin die Kochsalzrestriktion als eine wichtige nichtmedikamentöse Basis der Hochdruckbehandlung anzusehen haben.

Prof. Dr. Walter Zidek

Direktor der Medizinischen Klinik für Nephrologie,

Charité - Universitätsmedizin Berlin

\section{Interessenkonflikt}

Der Autor gibt an, dass kein Interessenkonflikt besteht. 